\title{
A Method of Driver Fatigue Detection based on Multi-features
}

\author{
Li Man and Meng Hui-ling \\ Engineering College, Xi'an International University, Xi'an, China \\ 48912111@qq.com
}

\begin{abstract}
Driver fatigue detection based on one feature is usually inaccurate. To solve the problem, a method of driver fatigue detection based on multi-features is proposed. We have analyzed the relationship between driver facial features, driving routes and vehicle handing behavior. Data of three features are collected by driving simulator and divided into two levels of awake and fatigue based on driver subjective assessment. After being discretized and normalized, the partial data is as the input data of neural network. The neural network is trained by BP algorithm. Other data is as the test samples. Experimental results show that compared with one feature, the method has higher accuracy rate and lower false positive rate.
\end{abstract}

Key words: driver; fatigue detection; multi-feature; sample; neural network

\section{Production}

Some data indicates that the traffic accidents caused by fatigue are increasing [1]. How to detect driver fatigue effectively has become a hot topic. Recently, the main methods include based on driver's physiological signal [2-4], based on driver's facial features [5-7], based on traffic characteristics [8-9] and based on vehicle handling behavior [10]. The method of based on driver's physiological signal has gotten the most research results which can use EEG, ECG, EMG, pulse and body fluid composition to estimate the fatigue status. It has high accuracy, but it also has invasiveness and it is difficult to promote to practice. The representative sample of based on driver's facial features is the PERCLOS (Percentage of Eyelid Closure over the Pupil over Time) and other samples are based on this standard. The method can realize online real-time and non-contact detection, but it is influenced by light and driver's own physiological characteristics. The methods of based on traffic characteristics and based on vehicle handling behavior are not affected by driver and environmental factors and the signal is easy to extract which can realize accurate measurement. But the correlation between traffic characteristics and vehicle handling behavior and the actual driver fatigue degree is difficult to quantify.

Based on the above analysis, we can conclude that single method can not detect driver fatigue state accurately. So we propose a method of driver fatigue detection based on multi-features. We analyze the relationship between driver facial features, driving routes and vehicle handing behavior. Data of three features are collected by driving simulator and divided into two levels of awake and fatigue based on driver subjective assessment. After being discretized and normalized, the partial data is as the input data of neural network. The neural network is trained by BP algorithm. Other data is as the test sample.

\section{Fatigue Detection System's Design}

The system's design contains driver's facial features detection and vehicle routes detection and vehicle handling behavior detection.

Driver's facial features detection includes P80 criterion and wink time and nod time. According to the PERCLOS, P80 criterion has the best correlation with driver's fatigue. 
And the time of wink can also be used to detect fatigue. Wink is a normal physiological reaction, but when driver is tired, wink will tend to be more frequent and the time is longer. Besides, nod is a feature parameter of driver fatigue detection. When driver is tired, the frequency of nod becomes more and the time becomes longer.

When driver is fatigue, there will appear distraction and be slow to respond to manipulate the vehicle which can lead to deviating from the driveway. Using a camera sensor, we can calculate the lane departure to judge the current driver's fatigue state.

Researches have found that when driver is fatigue, vehicle handling behavior will also change. For example, when driver is in the normal driving, they will turn the steering wheel slowly to keep vehicle along the lane. But when driver is fatigue, they will lose control of the steering wheel. Therefore, through the collection of steering signal can also realize the driver fatigue detection.

The proposed driver fatigue detection system structure is shown in figure 1.

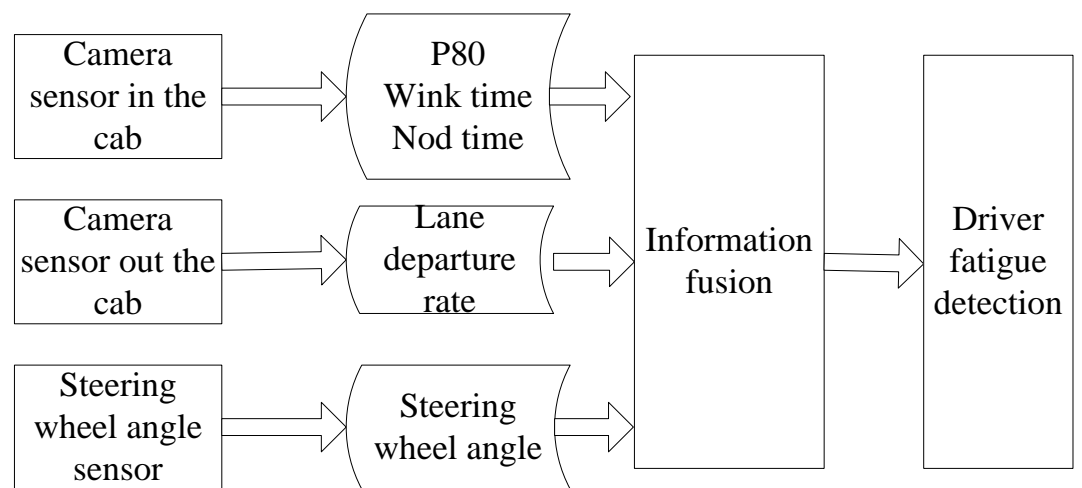

Figure 1. The System Construction Frame

\section{Fatigue Characteristic Parameters Detection and Analysis}

\subsection{Driving Simulation Experiments Design}

We use a driving simulator to simulate driving experiment. To remove the influence of different drivers' physiological characteristics, we select eight drivers (4 men and 4 women) to simulate driving experiment. Eight drivers all have more than 3 years driving experiences. Before experiments the sleep time of drivers is not less than $8 \mathrm{~h}$. Experimental periods are respectively at 00:00-4:00, 8:00-10:00, 13:00-15:00 and 19:00-21:00. At the same time, each driver fills out the form of self-evaluation: awake or fatigue every ten minutes.

\subsection{Facial Feature Parameters Inspection and Evaluation}

Driver's facial detection mainly refers to the features of eyes and mouth. We have proposed an improved Adaboost algorithm for eyes location. The algorithm uses five kinds of Haar-Like characteristics. The training samples of eyes are the eye images which are centered on the pupil and the upper boundary is brow. We use the method of cascade to train classifiers and each classifier can recognize most of eyes samples and reject most of non-eyes samples. In the first classifiers, the differences between eyes and non-eyes samples are evident and rectangular features are less so the first classifiers are simple. Along with the increase of the layers, classifiers gradually use more features and more complex structure to identify the samples.

Because mouth and skin have close color and mouth has less unique characteristics, the result of mouth location using Adaboost is poor. So we can locate mouth area roughly according to geometrical facial features. Mouth detection process is shown in figure 2. 


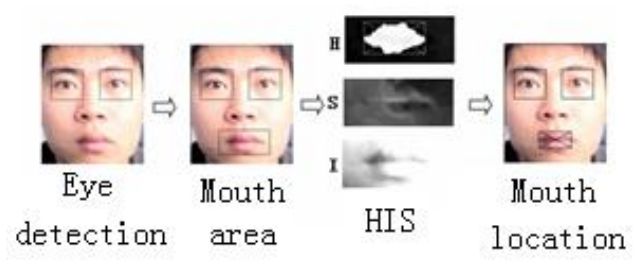

Figure 2. Mouth Detection Process

Eye state analysis means eye open detection. Eye area mainly includes sclera (white), iris and pupil (black). When eye is fully open, the area of iris and pupil is an ellipse which can be described as:

$$
S=\pi a b
$$

When eye is partly open, the area of black fleck is $S^{\prime}$. Eye open degree is described as:

$$
p=\frac{S^{\prime}}{S}
$$

In videos, the relative position of eyes and mouth will change when head posture changes and the change has some geometric features. Therefore, we can analyze head posture based on the geometric features. Nod means that head moves around $\mathrm{x}$ axis as shown in figure 3.

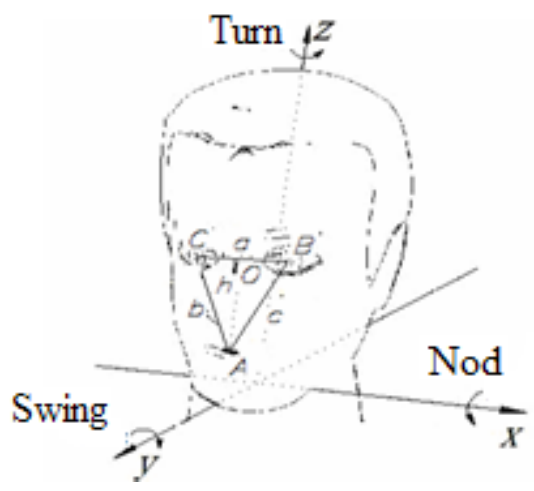

Figure 3. Head Model

Through the triangle property is unchanged, the height is changed. So nod angle $\beta$ can be represented as:

$$
\beta=\arccos \frac{h}{h^{\prime}}
$$

Among them, $h$ is the changed height; $h$ is the unchanged height of positive image.

By analyzing the experimental data, we have found that facial feature parameters have obvious differences between awake and fatigue. The differences are shown in table 1.

Table 1. The Differences between Awake and Fatigue

\begin{tabular}{cccc}
\hline State & P80/s & Wink/s & Nod/deg \\
\hline Awake & $<0.2$ & $<0.3$ & $<20$ \\
Fatigue & $\geqslant 0.2$ & $\geqslant 0.3$ & $\geqslant 20$ \\
\hline
\end{tabular}




\subsection{Lane Departure Rate Calculation and Analysis}

Firstly we should detect the lane. Algorithm has used a method based on inverse perspective mapping (IPM) and effective gradient. Firstly, road image is transformed into plan view using inverse perspective transform to eliminate perspective effect. Secondly, "Sobel" operator and morphological image processing are used to detect edge and eliminate noise. According to the actual lane width, effective gradient points are extracted. Finally, lane is fitted using least squares method. Lane detection process is shown in figure 4.

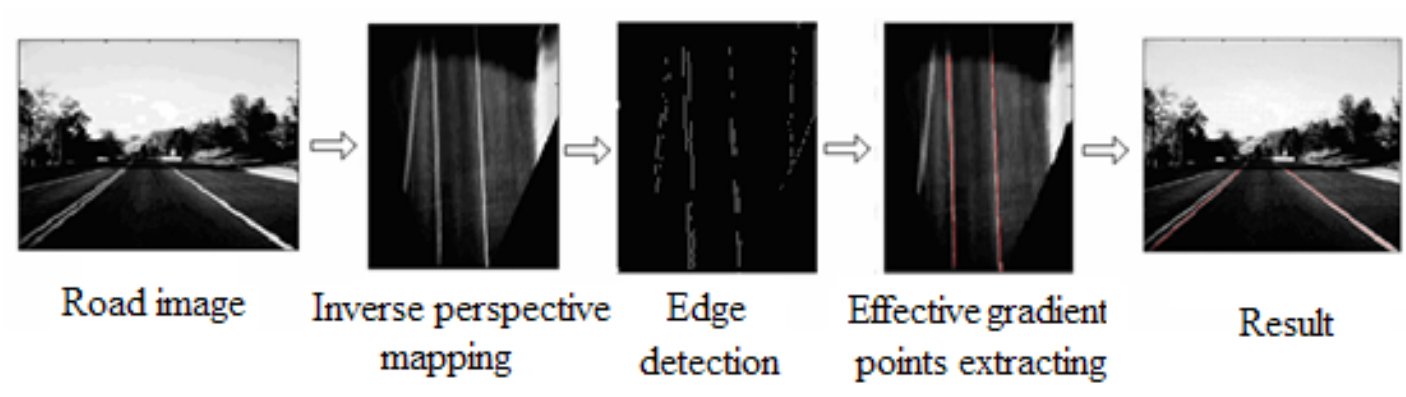

Figure 4. Lane Detection Process

According to the experimental data, lane departure rate of driver awake is stetted in the range of $[0.33 .0]$.

\subsection{The Steering Wheel Angle Acquisition and Analysis}

In the system, we have installed the steering wheel angle sensor to collect real-time signals. We have found that the means of steering wheel angle are close to 0 in the two state of awake and fatigue, but the variance is more different. The reason is that when driver is awake, he has enough energy to control the steering wheel changing slowly; but when driver is fatigue, he gradually lost control of the steering wheel so that the mutative extent gently increases. We have stetted the steering wheel angle variance of awake should be less than 2.5 degree.

\section{Neural Network Model for Fatigue Detection Design}

\subsection{Neural Network Structure}

Artificial neural network is a mathematical method which simulates the biological neural network to process information. The main work is to determine the layer number, node number, transfer function, the initial weights and bias, learning algorithm. BP neural network is a widely used method which usually includes an input layer, one hidden layer and one output layer. The number of input layer neurons is determined by the input feature vector. System chooses 5 input layer neurons. According to the approximate relationship between input layer and hidden layer, the number of hidden layer neurons is 11. The transfer function of hidden layer is s-shaped tansig. According to driver status, the number of output layer is 2. [ [ $\left.\begin{array}{ll}0 & 1\end{array}\right]$ represents awake and [ 10$]$ represents fatigue. The transfer function of output layer is linear purelin. The weight and bias of each node is based on the results of sample data training. The structure of BP neural network model is shown in figure 5. IW $\{1,1\}$ represents the weight of hidden layer; $b\{1\}$ represents the bias of hidden layer. $L W\{2,1\}$ represents the weight of output layer; $b\{2\}$ represents the bias of output layer. 


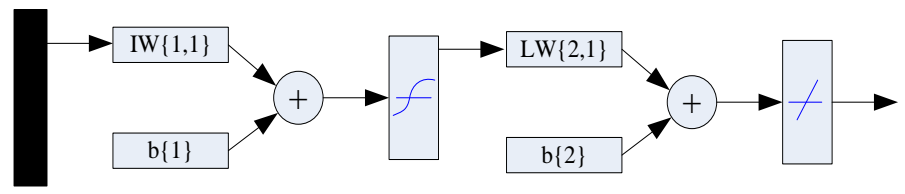

Figure 5. The Structure of BP Neural Network Model

\subsection{Neural Network Training}

To train the neural network model accurately, we have collected a large number of experimental data. The sample frequency is 10. After data being discretized and normalized, we have gotten 500 samples of two kinds of data finally. We have randomly selected 50 samples of awake data and fatigue data to train the neural network model. Neural network's learning rate is 0.1 ; the training target is 0.01 ; the initial weight and bias are the random values between $0 \sim 1$.

\section{Experiments and Analysis}

The rest of 450 samples are used to test the trained neural network and the results are shown in table 2 . We can conclude that the results of awake are close to $[0,1]$ and the results of fatigue are close to $[1,0]$. But there is also one sample (14) that can be not identified accurately.

Table 2. The Part Results of Samples being Identified based on Three Features

\begin{tabular}{cccc||cccc}
\hline Number & State & \multicolumn{2}{c||}{ Results } & Number & \multicolumn{2}{c}{ State } & \multicolumn{2}{c}{ Results } \\
\hline 1 & Awake & 1.0125 & 0.0032 & 11 & Fatigue & 0.0077 & 1.2305 \\
2 & Awake & 1.0042 & 0.0019 & 12 & Fatigue & 0.0129 & 1.0105 \\
3 & Awake & 0.9865 & 0.0189 & 13 & Fatigue & 0.0084 & 1.0414 \\
4 & Awake & 0.9683 & 0.0223 & 14 & Fatigue & 1.0426 & 0.0516 \\
5 & Awake & 1.0425 & 0.0631 & 15 & Fatigue & 0.0105 & 1.0206 \\
6 & Awake & 1.0012 & 0.0064 & 16 & Fatigue & 0.0001 & 1.0001 \\
7 & Awake & 1.0000 & 0.0000 & 17 & Fatigue & 0.0003 & 1.0000 \\
8 & Awake & 1.0001 & 0.0000 & 18 & Fatigue & 0.0001 & 1.0005 \\
9 & Awake & 1.0003 & 0.0001 & 19 & Fatigue & 0.0000 & 1.0000 \\
10 & Awake & 1.0000 & 0.0022 & 20 & Fatigue & 0.0001 & 1.0000 \\
\hline
\end{tabular}

The results of using a single feature are shown in table 3 . We conclude that the result of detecting steering wheel angle is better and the result of detecting facial features is not good. The reason is that the steering wheel angle detection is not affected by environmental factors and the facial features detection is relatively complex and greatly influenced by environmental factors and drivers themselves.

Table 3. The Results of Samples being Identified based on One Feature

\begin{tabular}{ccccccc}
\hline Samples/Group & \multicolumn{2}{c}{ Facial feature } & \multicolumn{2}{c}{ Lane departure rate } & \multicolumn{2}{c}{ Steering wheel angle } \\
\cline { 2 - 7 } & $\begin{array}{c}\text { Detection } \\
\text { rate } / \%\end{array}$ & $\begin{array}{c}\text { False } \\
\text { detection } \\
\text { rate/\% }\end{array}$ & $\begin{array}{c}\text { Detection } \\
\text { rate } / \%\end{array}$ & $\begin{array}{c}\text { False } \\
\text { detection } \\
\text { rate/\% }\end{array}$ & $\begin{array}{c}\text { Detection } \\
\text { rate /\% }\end{array}$ & $\begin{array}{c}\text { False } \\
\text { detection } \\
\text { rate/\% }\end{array}$ \\
\hline Awake (450) & 62.8 & 37.2 & 67.4 & 32.6 & 78.2 & 21.8 \\
Fatigue (450) & 57.6 & 42.4 & 76.5 & 23.5 & 82.7 & 17.3 \\
\hline
\end{tabular}


The results of using three features are shown in table 4 . The detection rate of awake samples is $93.6 \%$ and the false rate is $6.4 \%$. The detection rate of fatigue samples is $89.6 \%$ and the false rate is $10.4 \%$. Compared with using one single, using three features can greatly improve the accuracy of test results and reduce false detection.

Table 4. The Results of Samples being Identified based on Three Features

\begin{tabular}{ccccc}
\hline Samples/Group & \multicolumn{4}{c}{ Results } \\
\cline { 2 - 5 } & Awake/Group & Fatigue/Group & $\begin{array}{c}\text { Detection } \\
\text { rate/\% }\end{array}$ & $\begin{array}{c}\text { False detection } \\
\text { rate/\% }\end{array}$ \\
\hline Awake(450) & 421 & 29 & 93.6 & 6.4 \\
Fatigue (450) & 47 & 413 & 89.6 & 10.4 \\
\hline
\end{tabular}

\section{Conclusions}

We analyze the relationship between driver facial features, driving routes and vehicle handing behavior. Data of three features are collected by driving simulator and divided into two levels of fatigue based on driver subjective assessment. After being discretized and normalized, the partial data is as the input data of neural network. The neural network is trained by BP algorithm. Experimental results show that compared with one feature, the method has higher accuracy rate and lower false positive rate.

\section{References}

[1] W.-z. Zhang, Z.-c. Wang and Y.-x. Li, “An algorithm of color image enhancement for driver fatigue detection [J]", Journal of Optoelectronics, Laser, vol. 23, no. 8, (2012), pp. 1545-1549.

[2] S.-b. Wu, L. Gao and L.-a. Wang, "Detecting driving fatigue based on electroencephalogram [J]", Transactions of Beijing Institute of Technology, vol. 29, no. 12, (2011), pp. 1072-1075.

[3] S.-y. Hu and G.-t. Zheng, "Study on driver fatigue detection based on EEG spectrum-related features [J]", Journal of safety science and technology, vol. 6, no. 3, (2010), pp. 90-94.

[4] Y. Chen, Q. Huang and X. Liu, "All-weather detection method of driver fatigue [J]", Chinese Journal of Science Instrument, vol. 30, no. 3, (2009), pp. 636-637.

[5] T. Kinmura, K. Ishida and O. Noriyuki, "Feasibility study of sleepiness detection using expression features [J]", JSAE Review, no. 29, (2008), pp. 567-574.

[6] W. Zhang, Z. Wang and Y. Li, "Illumination compensation of color image for driver facial features detection [J]", International Journal of Digital Content Technology and its Applications, vol. 6, no. 17, (2012), pp. 475-482.

[7] W.-z. Zhang, Z.-c. Wang and Y.-x. Li, „Eye localization and state analysis for driver fatigue detection [J]”, Journal of Chongqing University, vol. 36, no. 1, (2013), pp. 22-28.

[8] V. Morellas, T. Morris and L. Alexander, "Preview Based Control of a Tractor Trailer Using DGPS for Preventing Road Departure Accidents [C]”, IEEE Conference on Intelligent Transportation System, ITSC 97, (1997) November, pp. 797-805.

[9] Z. Mao, X P. Yan and C Z. Wu, "Driving Fatigue Identification Method Based on Physiological Signals [C]", Proceedings of American Society of Civil Engineers. Presented on the 7th International Conference of Chinese Transportation Professionals, Shanghai, China, (2007), pp. 341-351.

[10] W. Li, Q.-c. He and X.-m. Fan, „Detection of driver's fatigue based on vehicle performance output [J]”, JOURNAL OF SHAN HAI JIAO TONG UNIVERSITY, vol. 44, no. 2, (2010), pp. 292-296.

[11] L. Bergasa and J. Nuevo, "Real-time system for monitoring driver vigilance [J]", IEEE Transactions on Intelligent Transportation Systems, vol. 7, no. 1 (2006), pp. 63-77.

[12] Y. Wang, X. Wang and C. Wen, „Gradient-pair constraint for structure lane detection [J]”, Journal of Image and Graphics, vol. 17, no. 6, (2012), pp. 657-663. 


\section{Authors}

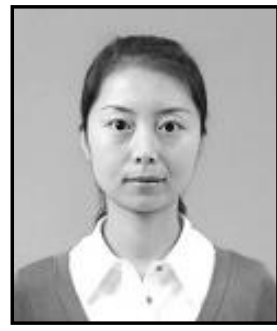

Li Man, she was born in 1980, is a teacher of Xi'an International University. Her main research field is the mechanical design and theory, control theory and control engineering. She has published several journal articles and undertook some scientific research projects.

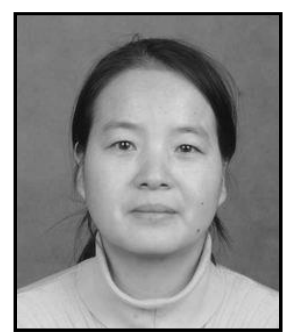

Meng Hui-ling, she who was born in 1978, has attained the degree of Master of mechanical and electronic engineering. Her main research field is the CAD technology on machinery. 
International Journal of Signal Processing, Image Processing and Pattern Recognition Vol.8, No.10 (2015) 